\title{
Older individuals have increased oro-nasal breathing during sleep
}

\author{
M.R. Madronio*, E. Di Somma*, R. Stavrinou*, J.P. Kirkness*,\#, E. Goldfinch*, J.R. Wheatley*,\#, \\ T.C. Amis*,\#
}

Older individuals have increased oro-nasal breathing during sleep. M.R. Madronio, E. Di Somma, R. Stavrinou, J.P. Kirkness, E. Goldfinch, J.R. Wheatley, T. C. Amis. (C) ERS Journals Ltd 2004.

ABSTRACT: Breathing route during sleep has been studied very little, however, it has potential importance in the pathophysiology of sleep disordered breathing.

Using overnight polysomnography, with separate nasal and oral thermocouple probes, data were obtained from 41 subjects (snorers and nonsnorers; 25 male and 16 female; aged 20-66 yrs). Awake, upright, inspiratory nasal resistance (Rn) was measured using posterior rhinomanometry. Each 30-s sleep epoch (not affected by apnoeas/hypopnoeas) was scored for presence of nasal and/or oral breathing.

Overnight, seven subjects breathed nasally, one subject oro-nasally and the remainder switched between nasal and oro-nasal breathing. Oral-only breathing rarely occurred. Nasal breathing epochs were 55.79 (69.78) per cent of total sleep epochs (\%TSE; median (interquartile range)), a value not significantly different to that for oro-nasal (TSE: $44.21(68.66) \%)$. Oro-nasal breathing was not related to snoring, sleep stage, posture, body mass index, height, weight, $\mathrm{Rn}\left(2.19(1.77) \mathrm{cm} \mathrm{H}_{2} \mathrm{O} \cdot \mathrm{L}^{-1} \cdot \mathrm{sec}^{-1}\right)$ or sex, but was positively associated with age. Subjects $\geqslant 40$ yrs were approximately six times more likely than younger subjects to spend $>\mathbf{5 0} \%$ of sleep epochs utilising oro-nasal breathing.

Ageing is associated with an increasing occurrence of oro-nasal breathing during sleep.

Eur Respir J 2004; 24: 71-77.
*Ludwig Engel Centre for Respiratory Research, Dept of Respiratory Medicine, Westmead Hospital, and "University of Sydney, New South Wales, Australia.

Correspondence: T.C. Amis, Ludwig Engel Centre for Respiratory Research, Westmead Hospital, Westmead, New South Wales, 2145 Australia.

Fax: 61298457286

E-mail: terencea@westgate.wh.usyd.edu.au

Keywords: Ageing

breathing route

sleep

Received: January 142003

Accepted after revision: January 242004

The study was supported by the National Health and Medical Research Council of Australia
During wakefulness, changes in breathing route (i.e. nasal versus oral breathing) are most notably associated with oral augmentation of breathing during exercise. However, breathing via the nose or mouth, or a combination of both, is also encountered during sleep [1]. Furthermore, the breathing route may be a contributing factor in the occurrence of sleep disordered breathing (SDB). For example, it has been reported that subjects with more mouth breathing during sleep tend to experience more episodes of apnoea than those with predominantly nasal breathing [1]. In addition, nasal obstruction, which may predispose to mouth breathing, has also been linked to SDB [2]. Theoretically, a high nasal resistance should be associated with lower intraluminal pressures during inspiration, thus favouring upper airway narrowing and/or collapse. Switching breathing route from nasal to oral or oro-nasal may help to ameliorate this effect since, depending on the degree the mouth is opened, oral airway resistance can be lowered to values well below that of the nasal passages [3]. However, a switch to oral route breathing may, in itself, predispose the upper airway to collapse since a number of upper airway muscles have route-dependent respiratory-related activity [4-6]. MEURICE et al. [7] have also reported that opening the mouth during sleep increases upper airway collapsibility, while breathing through the mouth also has the potential to dry the upper airway mucosa, enhancing collapsibility and increasing adhesive effects [8].

There have been only two previous quantitative studies of breathing route during sleep, both using a partitioned face mask and pneumotachographs. In a study of 14 subjects, GLEESON et al. [1] found that, during sleep, older males breathed a greater proportion of total ventilation via the mouth than females and younger males. More recently, FITZPATRICK et al. [9] studied 10 healthy subjects (all with normal levels of awake nasal resistance values) and found an inhaled ventilation oral fraction of $\sim 4 \%$ during sleep. In the present study, the authors aimed to document breathing route usage during sleep in a larger group of nonsnorers and snorers of both sexes and to examine the relationship between breathing route during sleep and a range of anthropometric and sleep-related parameters.

\section{Materials and methods}

\section{Study subjects}

Breathing route during sleep was monitored in 43 adult subjects who volunteered for polysomnographic studies of breathing and/or snoring during sleep. In the course of the study, two subjects were diagnosed with obstructive sleep apnoea and were excluded, leaving 41 subjects for analysis. Seventeen of these subjects reported a history of habitual snoring. Anthropometric data, including age, height, weight, body mass index (BMI), ethnic origin and sex were obtained for all subjects. The study was approved by the Western Sydney Area Health Service Human Ethics Committee.

\section{Study design}

Breathing route data during sleep were obtained for each subject from a single overnight polysomnographic study 
performed in a sleep laboratory (refer to Polysomnography section). Inspiratory nasal airflow resistance ( $\mathrm{Rn}: 0.4 \mathrm{~L} \cdot \mathrm{sec}^{-1}$ ) in the upright seated posture was also measured during wakefulness in each subject within $24 \mathrm{~h}$ of the sleep study (refer to Nasal resistance section). Relationships between the occurrence of nasal, oral or oro-nasal breathing during sleep and anthropometric factors, $\mathrm{Rn}$ and polysomnographic variables were then examined using statistical modelling techniques (refer to Data analysis section).

\section{Polysomnography}

Routine sleep variables were recorded using standard polysomnography. Data recorded included: electro-encephalogram; electrocardiogram; electrooculogram; submental and diaphragmatical electromyograms; arterial oxygen saturation; impedance plethysmography (P/N 7000-0049-00; Compumedics, Abbotsford, Australia); body position; and nasal pressure (1600 Nasal Cannula; Salter Labs Inc., Arvin, CA, USA). The tip of the nasal cannula was modified for each study by clipping $3 \mathrm{~mm}$ from the tip to increase the crosssectional area for detection of nasal pressure. The nasal cannula was inserted in the opening of the nostrils.

Breathing route was recorded using a dual-channel nasal/ oral thermocouple (F-ONT2A; Grass, West Warwick RI, USA). The thermocouple was placed on top of the nasal cannula and both were taped to the sides of the face to minimise movement during sleep. Snoring was recorded using either a microphone (NL-05 Type 2; RION Co., Ltd, Tokyo, Japan) suspended $20 \mathrm{~cm}$ directly above the subject's mouth or an external tracheal microphone (P/N 7002-0017-03; Compumedics) attached directly to the right side of the subject's neck. All monitored parameters were recorded directly on a computer using proprietary software (W Series, V2; Compumedics). There was no cross-contamination between the oral and nasal thermocouple signals, as confirmed by having each subject perform short periods (five breaths) of exclusive nasal and exclusive oral breathing in the supine and right lateral postures immediately prior to lights out.

\section{Nasal resistance}

For each subject, Rn was measured during wakefulness in the upright, seated posture using standard posterior rhinomanometry [10] within $24 \mathrm{~h}$ of polysomnography.

\section{Data analysis}

Each sleep study was sleep staged for 30-s epochs using RECHSHTAFFEN and KALES [11] criteria. Respiratory events (respiratory disturbance index (RDI): number of apnoeas plus the number of hypopnoeas per hour of sleep) were identified using American Academy of Sleep Medicine criteria [12]. Epochs containing such events, as well as arousals, movements or signal artefacts were excluded from analysis $(0-14 \%$ of total epochs for individuals). Route of breathing was assessed by using the nasal and oral thermocouple signals to classify each 30 -s epoch as either a nasal, oral or oro-nasal breathing epoch. Nasal breathing epochs were defined as epochs containing $\geqslant 3$ consecutive phasic signals on the nasal thermocouple channel only. Oral breathing epochs were defined as epochs containing $\geqslant 3$ consecutive phasic signals on the oral thermocouple channel only. Oro-nasal breathing epochs contained $\geqslant 3$ consecutive phase linked signals on both the nasal and oral thermocouple channels. Each epoch was also classified as either a nonsnoring epoch or a snoring epoch. Snoring epochs were defined as epochs containing $\geqslant 3$ consecutive sound peaks in phase with inspiration (determined using the P-nasal signal) and with a peak amplitude of $\geqslant 5 \mathrm{~dB}$ above the background sound level ( $\sim 39-50 \mathrm{~dB}$ for individual study nights).

The occurrence of nasal breathing, oral breathing, oronasal breathing and snoring epochs was expressed as a percentage of the total sleep epochs (TSE) analysed. Similar data were generated for sleep stage and body position. Data for left and right lateral decubitus were combined. Minimal data were obtained for the prone posture, consequently, this body position was not included in the analysis.

Frequency histograms were developed for each measured parameter. Data were also expressed as a median and interquartile range (IR). Very little oral-only breathing (0-0.04\% TSE for individuals; refer to Results section) was detected, consequently, these data were not included in any further analyses. Data were compared using the Wilcoxon signed rank test. Analysis of the relationship between breathing route and other measured parameters was expressed in terms of their association with the occurrence of oro-nasal breathing epochs (\%TSE). Because of the relative absence of oral-only breathing, results for nasal-only breathing were the reverse of those for oro-nasal breathing. Statistical modelling was performed in a three-stage procedure. First, correlative analyses (Pearson's correlation coefficient, r) were performed to search for significant univariate relationships. Stepwise multivariate regression analysis was then used to test for independent relationships. Logistic regression analysis was also used to examine the extent to which age $\geqslant 40$ yrs predicted the occurrence of oronasal breathing epochs during sleep. A p-value of $<0.05$ was considered significant.

\section{Results}

\section{Anthropometric data}

Figure 1a-d summarises the anthropometric characteristics of the study cohort that contained 35 persons of White ethnic origin, six persons of Asian ethnic origin, 25 males and 16 females.

\section{Nasal resistance}

For the group ( $\mathrm{n}=39)$, awake inspiratory $\mathrm{Rn}$ was 2.2 (1.8) $\mathrm{cm} \mathrm{H}_{2} \mathrm{O} \cdot \mathrm{L}^{-1} \cdot \mathrm{sec}^{-1}$ with values ranging $0.6-6.8 \mathrm{~cm}$ $\mathrm{H}_{2} \mathrm{O} \cdot \mathrm{L}^{-1} \cdot \mathrm{sec}^{-1}$ (fig. 1e).

\section{Polysomnographic variables}

Frequency distribution histograms for polysomnographic variables versus number of subjects are shown in figure $1 \mathrm{f}-1$. Most subjects slept reasonably well, with total sleep time ranging 148.5-386.5 min. For the group, 2.9 (4.4) \% TSE was spent in nonrepetative eye movement (NREM) Stage I sleep; 57.9 (18.0) \% TSE in NREM Stage II; 12.6 (7.8) \% TSE in NREM Stage III; 6.4 (13.2) \%TSE in NREM Stage IV; and 15.3 (10.5) \% TSE in repetitive eye movement sleep. Sleep time was equally divided between the supine (45.9 (45.3) \%TSE) and lateral body positions (41.2 (48.0) \% TSE; $\mathrm{p}=0.51)$. Snoring sounds were detected in 27 subjects (fig. 11). All subjects had an RDI $<10 \cdot \mathrm{h}^{-1}$. 

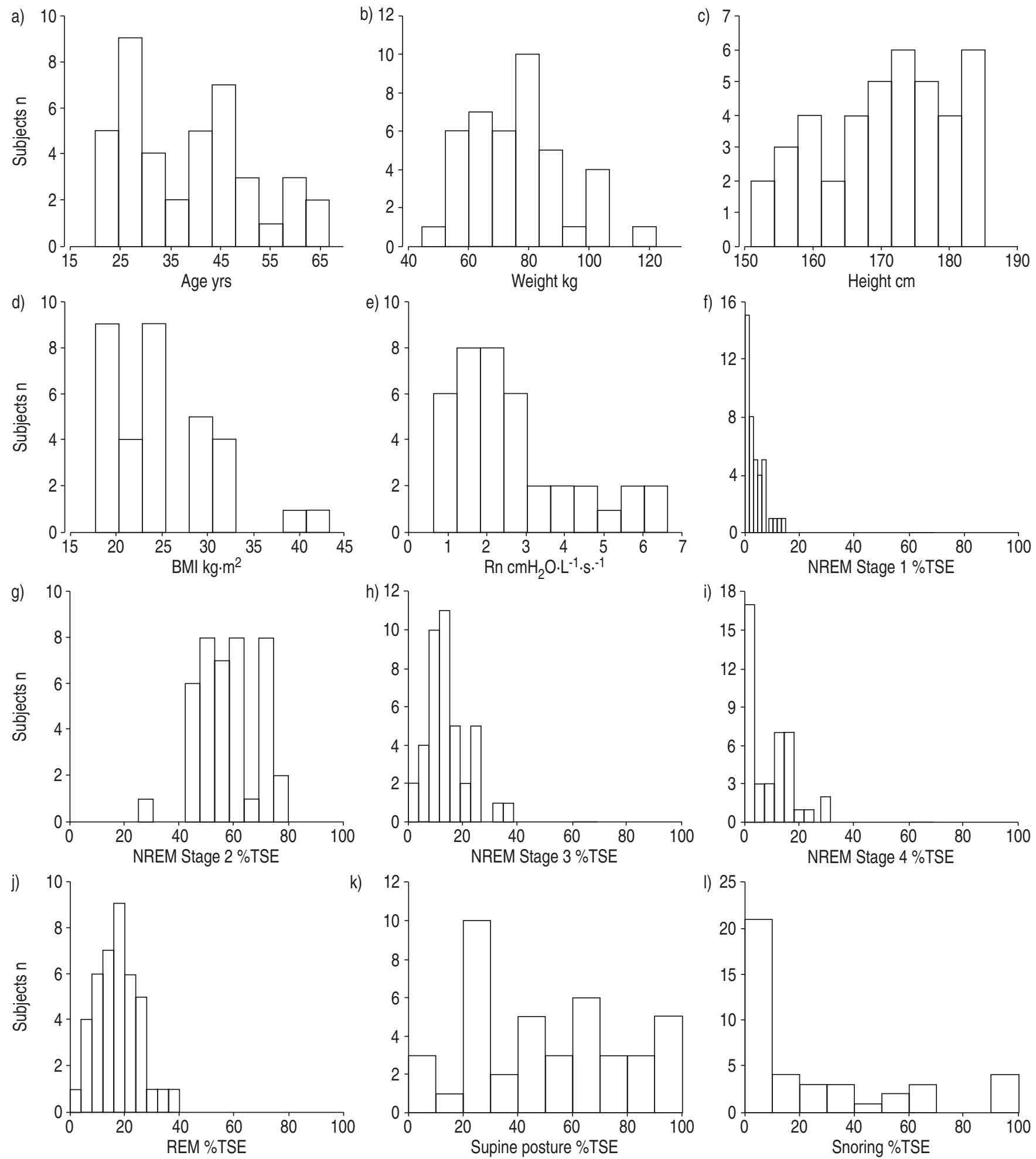

Fig. 1.-Frequency distribution histograms in 41 subjects for a-d) anthropometric factors, e) nasal resistance, $\mathrm{f}-\mathrm{j}$ ) sleep architecture, $\mathrm{k}$ ) supine sleep posture and 1) occurrence of snoring epochs. Note the frequency distribution histogram for lateral posture is the exact reverse of that for the supine posture and is not shown. TSE: total sleep epochs; Rn: nasal resistance at $0.4 \mathrm{~L} \cdot \mathrm{s}^{-1}$; BMI: body mass index; REM: rapid eye movement sleep; NREM: nonrapid eye movement sleep.

\section{Breathing route during sleep}

Figure 2 shows the raw data demonstrating nasal and oronasal breathing signals during different periods of NREM sleep in one subject. Figure $3 a-c$ summarises the occurrence of nasal, oral and oro-nasal breathing epochs for the group.
During sleep, 11 subjects breathed exclusively or almost exclusively via the nasal route throughout the night, while six subjects breathed exclusively or almost exclusively oro-nasally all night. The remaining subjects switched their breathing route between nasal-only breathing and oro-nasal breathing throughout the night. There was no fixed pattern associated 

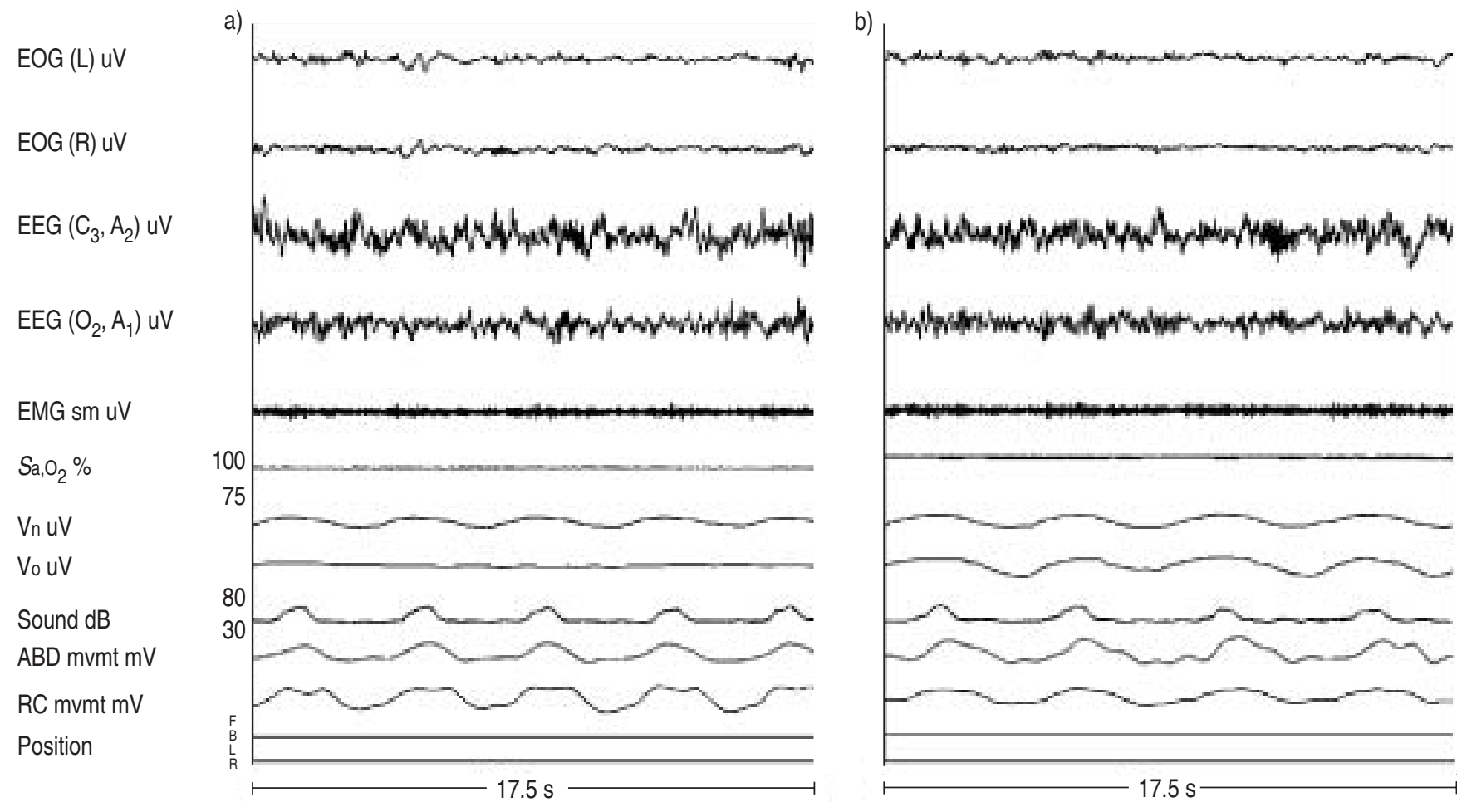

Fig. 2. - Raw data recorded during nonrapid eye movement sleep in the supine posture in one subject during a) nasal-only breathing and during b) oro-nasal breathing. Note that snoring occurs during both nasal-only breathing and oro-nasal breathing. EOG: electrooculogram; L: left; R: right; EEG: electroencephalogram; EMG sm: submental electromyogram; $\mathrm{Sa}_{\mathrm{a}} \mathrm{O}_{2}$ : arterial oxygen saturation; Vn: nasal airflow; Vo: oral airflow (thermocouple); Sound: microphone; ABD mvmt: abdominal movement (impedance plethysmography); RC mvmt: chest movement (impedance plethysmography); Position: body position; F: front; B: back. Inspiration is upwards in Vn, Vo, ABD and RC mvmt. EEG electrodes named according to position: C: central; O: occipital; A: auricular; even number: right side; odd number: left side.

with breathing route changes with considerable intra- and inter-subject variability in the duration (every few minutes to $\sim 20-40$-min intervals) of nasal or oro-nasal breathing periods before a switch to the alternate pattern was initiated. Exclusive oral breathing was rare, occurring in only three subjects (0.003-0.04\%TSE). Indeed, of the 26,498 epochs classified for breathing route, only 27 of these were oral-only breathing epochs. For the group, the occurrence of nasal breathing (55.8 (69.8) \% TSE) was not significantly different to that for oro-nasal breathing (44.21 (68.66) \% TSE; $p=0.14)$.

\section{Breathing route during snoring}

Both nasal and oro-nasal breathing occurred during snoring epochs (fig. 2), however, the relative occurrence rates varied widely for individuals. For the group, 56.5 (80.7) $\%$ of snoring epochs were also oro-nasal breathing epochs.

\section{Univariate analysis}

When the data were examined for univariate relationships, the occurrence of oro-nasal breathing epochs during sleep (i.e. oro-nasal breathing epochs \%TSE) correlated positively with age $(\mathrm{r}=0.42 ; \mathrm{p}<0.01)$ and $\mathrm{BMI}(\mathrm{r}=0.33 ; \mathrm{p}=0.03)$, tended to correlate positively with the occurrence of snoring epochs $(\mathrm{r}=0.29 ; \mathrm{p}=0.06)$, and correlated negatively with the occurrence of nonsnoring epochs $(\mathrm{r}=-0.34 ; \mathrm{p}=0.03)$.

\section{Multivariate regression analysis}

Stepwise multivariate regression analysis revealed that the only independent predictor for the occurrence of oro-nasal breathing epochs during sleep was age (oro-nasal breathing epochs: \%TSE $=1.17$ yrs -2.71 ; adjusted $r^{2}=0.15 ; \quad p<0.01$; fig. 4).

\section{Logistic regression analysis}

Table 1 shows the occurrence of oro-nasal breathing epochs $>$ and $<50 \%$ TSE for subjects with age $\geqslant 40$ yrs and $<40$ yrs. Logistic regression analysis confirmed that an age of $\geqslant 40 \mathrm{yrs}$ was a significant predictor for an occurrence of $>50 \% \mathrm{TSE}$ for oro-nasal breathing epochs during sleep ( $\mathrm{p}=0.01$; odds ratio $=5.8 ; 95 \% \mathrm{CI}: 1.5-22$ ).

\section{Discussion}

The major finding in this study was that snorers and nonsnorers utilised both nasal and oro-nasal breathing during sleep. Furthermore, most subjects used nasal breathing throughout the night, but added oral breathing intermittently, such that, for the group, the occurrence of oro-nasal breathing sleep epochs was not significantly different to that for nasal-only breathing. Pure oral breathing during sleep occurred rarely. Snoring occurred with both nasal and oronasal breathing. Age was the only independent predictor for 

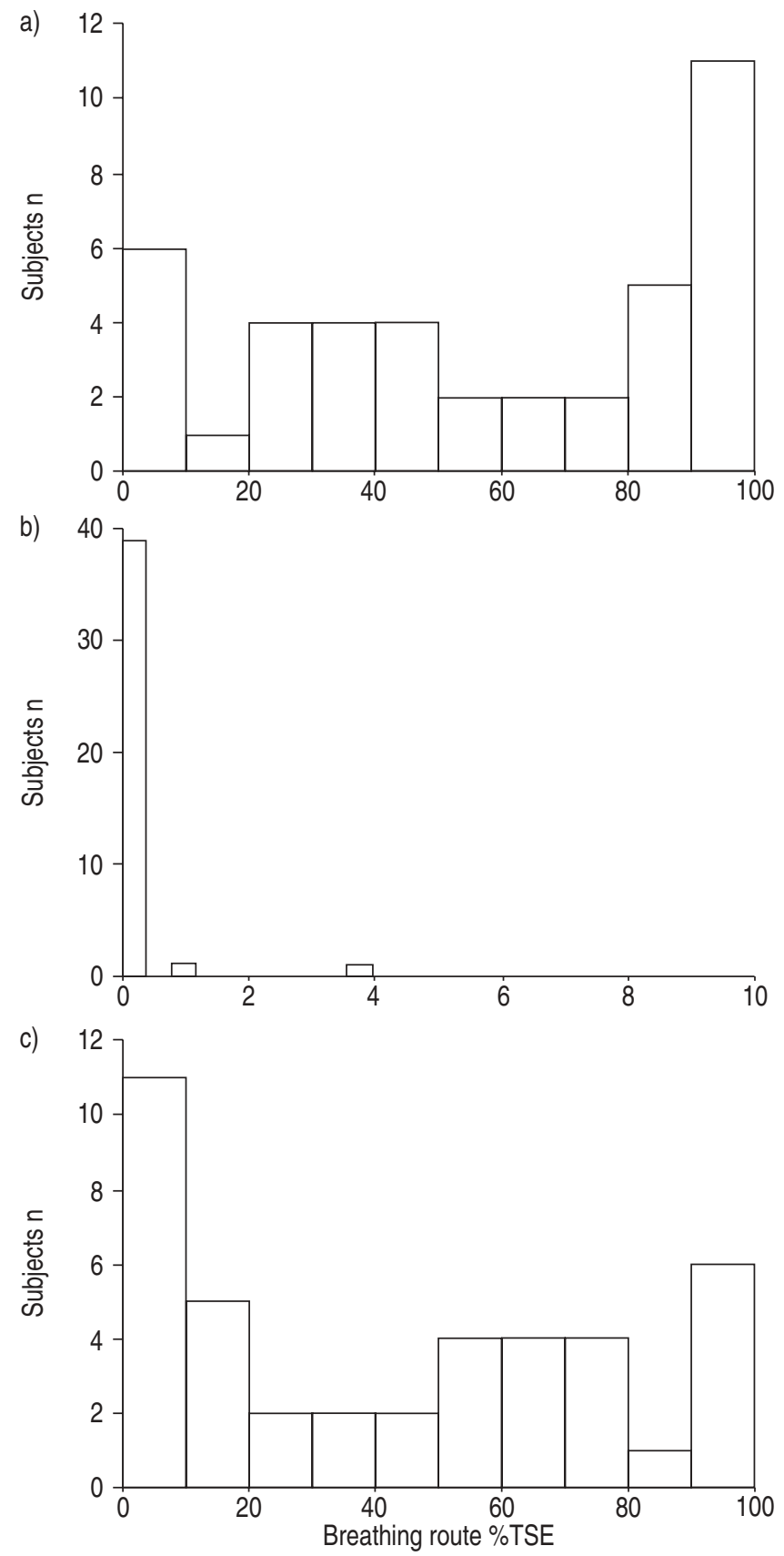

Fig. 3. - Frequency distribution histograms for a) occurrence of nasalonly, b) oral-only and c) oro-nasal breathing epochs during sleep in 41 subjects. TSE: total sleep epochs.

breathing route during sleep. Subjects $\geqslant 40$ yrs of age were approximately six times more likely than younger subjects to spend $>50 \%$ of the night utilising oro-nasal breathing.

The authors monitored breathing routes using a dual channel thermocouple device that sensed nasal and oral airflow separately. Such devices have been used extensively for monitoring airflow during sleep [13-15]. Potential disadvantages associated with the use of these devices include failure to sample airflow adequately because of inappropriate positioning of sensors or dislodgment during sleep, crosscontamination from the alternate pathway, and poor sensitivity to changes in airflow because of nonlinear response characteristics [15]. In the present study, the thermocouple leads were supported by hooking them behind the ears and

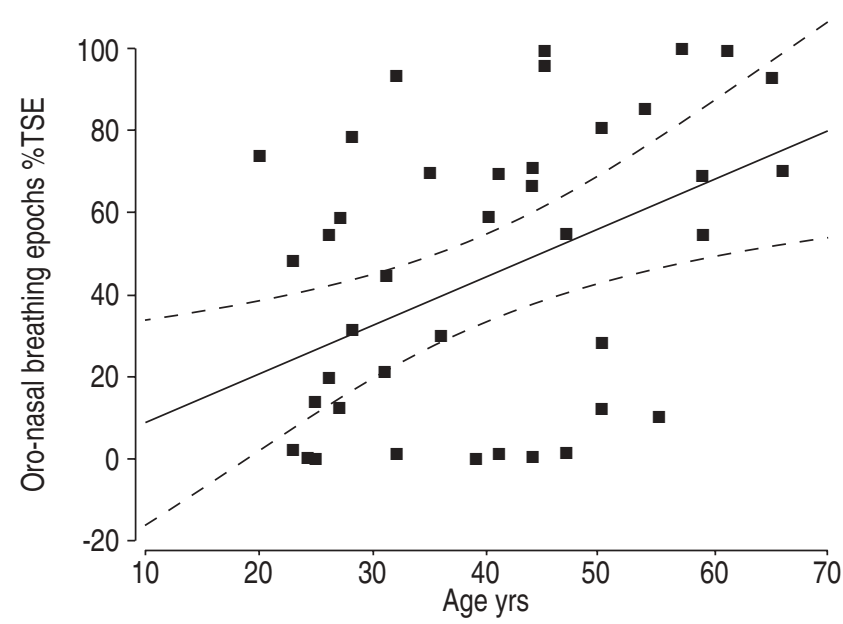

Fig. 4.-Relationship between age and occurrence of oro-nasal breathing epochs during sleep. — : regression line; - - -: 95\% CI limits for prediction equation.

taping them to the subject's cheeks. The absence of crosscontamination was verified at the commencement of each study by having the subject voluntarily breathe via the nasal and oral routes separately and ensuring that no signal was detected for the opposite route. Changes in sensor position during the night, however, remain a potential complication.

It is well established that thermocouple devices have limitations for the detection of airflow because of their nonlinear response characteristics [12]. However, the principal problem here is poor sensitivity to changes in airflow at higher airflows [15]. In fact, these devices have high sensitivity for detecting the presence (or absence) of airflow [14]. Thermocouple signals were used in the present study only for this latter purpose and no attempt was made to quantitate the level of airflow present. Thermal-based devices for the detection of respiratory airflow sense changes in temperature associated with exposure to inhaled or exhaled gas. Therefore, should inspiration and expiration take place via different breathing routes the interpretation of a thermocouple signal as representing breathing via a single route may be confounded. Thus, in the context of the present study, breathing patterns that involved, for example, inhalation via the nose and exhalation via the mouth, would be classified as oro-nasal breathing if a nasal and oral route signal was present or oral only if there were only an oral signal. This latter possibility is unlikely since oral-only breathing was encountered rarely. The former pattern, however, would have been included in the present occurrence rate for oro-nasal breathing.

Instrumentation with nasal cannulae has been shown to increase nasal resistance in some individuals, particularly those with a high baseline nasal resistance [16]. Consequently, it is possible that the breathing route in the present study was

Table 1. - Subject age and occurrence of oro-nasal breathing epochs during sleep

\begin{tabular}{lcc}
\hline Oro-nasal breathing epochs \%TSE & \multicolumn{2}{c}{ Number of subjects n } \\
\cline { 2 - 3 } & Age $<40$ yrs & Age $\geqslant 40$ yrs \\
\hline $550 \%$ & 16 & 6 \\
$>50 \%$ & 6 & 13 \\
Total number of subjects & 22 & 19 \\
\hline
\end{tabular}

\%TSE: per cent total sleep epochs. 
influenced by the presence of the nasal pressure cannulae and thermocouple probes. However, this would seem unlikely in the current study's subject group since baseline nasal resistance did not emerge as a significant predictor of breathing route during sleep.

The present authors found that most subjects used the nasal route for breathing while asleep, either exclusively or in combination with oral breathing. These findings are in general agreement with other studies on this topic [1,9], using a dual compartment face mask with attached pneumotachographs to monitor nasal and oral airflow during sleep. While the authors have demonstrated an $44 \%$ TSE occurrence of oro-nasal breathing during sleep, findings reported by FITZPATRICK et al. [9] suggest that the magnitude of the oral fraction of minute ventilation associated with this pattern of breathing may be relatively small ( 4 \%), i.e. although oronasal breathing occurs frequently during sleep most of the minute ventilation remains via the nasal route. In the study by GLEESON et al. [1] they found that nasal breathing always contributed a minimum of one third of the total ventilation during sleep but the contribution of oral breathing varied. GLEESON et al. [1] did not observe any oral-only breathing. Since their subjects were encumbered by a face mask the issue remains as to the extent to which this instrumentation altered the breathing route. However, the present study has now demonstrated a similar finding based on the occurrence of nasal and oro-nasal breathing epochs, and achieved this without instrumentation of the face, beyond the attachment of sensors to the skin.

The current authors were interested in examining what factors might be associated with the oral augmentation of nasal breathing during sleep and, therefore, analysed the current data for significant associations between both anthropometrical and polysomnographical variables, and the occurrence of oro-nasal breathing epochs. Positive univariate relationships were found between oro-nasal breathing epochs during sleep and both age and BMI, while a negative association with nonsnoring epochs, and a borderline significant positive association with snoring epochs, were also demonstrated. Weight and height, each considered separately, and ethnic origin (White or Asian), together with sleep stage and sleeping posture (back versus side) all showed no association with breathing route. Multivariate regression analysis demonstrated that age was the only identified independent predictor of oro-nasal breathing during sleep. The univariate associations with BMI and nonsnoring reflected the tendency for older individuals in the current study's cohort to have higher levels of BMI and for younger individuals not to snore. The association between age and oro-nasal breathing explained only $\sim 15 \%$ of the variance. Consequently, while there was a significant positive association between age and oro-nasal breathing during sleep, the effect was relatively weak and clearly there must be other influences that were not measured in the present study. Nevertheless, the relationship demonstrated in fig. 4 predicts an increase in the occurrence of oronasal breathing epochs during sleep of $\sim 1 \%$ TSE for each age year.

In the study by GLEESON et al. [1], a much stronger positive correlation $(\mathrm{r}=0.83)$ between oral breathing during sleep and age in males only was found. While differences in correlation strength between the two studies might reflect differences in study size and subject characteristics, in the present study sex was not associated with breathing route during sleep. This discrepancy between the two studies may relate to the fact that the study by GLEESON et al. [1] contained only seven males and seven females and had a wider range of ages and more individuals $>40$ yrs of age in the male group than in the female group. Since multivariate analysis was not performed in the study by GLEESON et al. [1] this finding may, in fact, reflect an age, rather than a sex effect.
The mechanisms linking age and oro-nasal breathing during sleep are not known. GLEESON et al. [1] suggested that the effect may be related to an increased $\mathrm{Rn}$ in males. However, in the present study there was no association between awake Rn values and breathing route during sleep. The median value for inspiratory $\mathrm{Rn}$ in the present study group was $2.19 \mathrm{~cm} \mathrm{H} \mathrm{H}_{2} \mathrm{O} \cdot \mathrm{L}^{-1} \cdot \mathrm{sec}^{-1}$, a value within the normal range for the current authors' laboratory. The lack of a relationship between $\mathrm{Rn}$ and breathing route would, however, not be likely to apply at high $\mathrm{Rn}$ levels. In addition, $\mathrm{Rn}$ in the upright posture and during wakefulness was measured and this value may not be reflective of that occurring in the supine posture and during sleep. However, for subjects with a relatively normal $\mathrm{Rn}$, breathing route during sleep is not predicted by awake upright $\mathrm{Rn}$. Therefore, $\mathrm{Rn}$ does not appear to explain the relationship between breathing route and age, and other mechanisms must play a role. Such effects might include ageing effects on jaw and upper airway muscle activity during sleep and/or loss of skeletal muscle strength. It should be noted, however, that the relationship is weak and clearly there are other factors involved that are not (at least not tightly) related to age.

Mouth opening during sleep has been associated with increased upper airway collapsibility even in healthy subjects [7]. This effect is thought to be mediated via a combination of upper airway narrowing and decreased efficiency of upper airway dilator muscle action [7]. In the present study the occurrence of snoring epochs tended to correlate with the occurrence of oro-nasal breathing epochs, while there was a negative relationship between nonsnoring epochs and oronasal breathing (i.e. absence of snoring was associated with the absence of oro-nasal breathing). However, both these relationships were not significant when corrected for the effect of age and, thus, probably reflect a tendency for the snorers to be older than the nonsnorers.

Mouth breathing and snoring have been previously linked temporally and an association between the two postulated [1]. However, it has also been recognised for some time that both nasal and oro-nasal snores occur [17]. The present study suggests that oro-nasal breathing is not a prerequisite for snoring and that while the occurrence of oro-nasal and nasal snoring varies widely between individuals, on a group basis, nasal and oro-nasal snoring occur with approximately equal frequency.

The presence of snoring in the current study's subjects suggests that some breaths may have been characterised by inspiratory flow limitation (IFL) and increased upper airway resistance (UAR). The extent to which this occurred was not assessed in the present study. However, the lack of a demonstrated relationship between snoring and breathing route makes it unlikely that IFL or UAR influenced breathing route in the study's subjects since the snoring epochs are more likely to be characterised by IFL and increased UAR.

This study, along with previous investigations [9, 16], demonstrates that the oral breathing route is utilised during sleep in some subjects. Consequently, the use of nasal breathing monitoring (e.g. P-nasal) alone to monitor sleep disordered breathing may be potentially confounded by the occurrence of oral route breathing.

To summarise, the authors demonstrated that snoring and nonsnoring subjects utilise both nasal and oro-nasal, but rarely oral-only, breathing during sleep. The occurrence of oro-nasal breathing sleep epochs increases with increasing age and is not related to: sex; White or Asian ethnic origin; height; weight; body mass index; awake nasal resistance (within the normal range); sleep stage; sleeping position or the occurrence of snoring. The authors conclude that ageing is associated with an increased oro- nasal breathing during sleep. 
Acknowledgements. The authors wish to acknowledge the invaluable assistance and advice of $\mathrm{K}$. Byth in the statistical analysis of the data.

\section{References}

1. Gleeson K, Zwillich CW, Braier K, White DP. Breathing route during sleep. Am Rev Respir Dis 1986; 134: 115-120.

2. Scharf MB, Cohen AP. Diagnostic and treatment implications of nasal obstruction in snoring and obstructive sleep apnea. Ann Allergy Asthma Immunol 1998; 81: 279-290.

3. Amis TC, $\mathrm{O}^{\prime}$ Neill $\mathrm{N}$, Wheatley JR. Oral airway flow dynamics in healthy humans. J Physiol 1999; 515: 293-298.

4. Basner RC, Simon PM, Schwartzstein RM, Weinberger SE, Weiss JW. Breathing route influences upper airway muscle activity in awake normal adults. J Appl Physiol 1989; 66: 1766-1771.

5. Tangel DJ, Mezzanotte WS, White DP. Influences of NREM sleep on activity of palatoglossus and levator palatini muscles in normal men. J Appl Physiol 1995; 78: 689-695.

6. Mortimore IL, Douglas NJ. Palatopharyngeus has respiratory activity and responds to negative pressure in sleep apnoeics. Eur Respir J 1996; 9: 773-778.

7. Meurice JC, Marc I, Carrier G, Series F. Effects of mouth opening on upper airway collapsibility in normal sleeping subjects. Am J Respir Crit Care Med 1996; 153: 255-259.

8. Olsen LG, Strohl KP. Airway secretions influence upper airway patency in the rabbit. Am Rev Respir Dis 1988; 137: 1379-1381.

9. Fitzpatrick MF, Driver HS, Chatha N, Voduc N, Girard AM.
Partitioning of inhaled ventilation between the nasal and oral routes during sleep in normal subjects. J Appl Physiol 2003; 94: 883-890.

10. Wheatley JR, Amis TC, Engel LA. Nasal and oral airway pressure-flow relationships. J Appl Physiol 1991; 71: 23172324.

11. Rechtschaffen A, Kales, A, eds. A Manual of Standardized Terminology, Techniques and Scoring System for Sleep Stages of Human Subjects. Publication No. 204. Bethesda, National Institutes of Health, 1968.

12. American Academy of Sleep Medicine Task Force. Sleeprelated breathing disorders in adults: recommendations for syndrome definition and measurement techniques in clinical research. Sleep 1999; 22: 667-689.

13. Pennock BE. Quantitative measurement of ventilation at the mouth and nose with heat sensors. Bio Med Instr Tech 1992; 26: $492-502$.

14. Norman RG, Ahmed MM, Walsleben JA, Rapoport DM. Detection of respiratory events during NPSG; nasal cannula/pressure sensor versus thermistor. Sleep 1997; 20: $1175-1184$.

15. Farre R, Montserrat JM, Rotger M, Ballester E, Navajas D. Accuracy of thermistors and thermocouples as flowmeasuring devices for detecting hypopnoeas. Eur Respir $J$ 1998; 11: 179-182.

16. Lorino A-M, Lorino H, Dahan E, et al. Effects of nasal prongs on nasal airflow resistance. Chest 2000; 118: 366-371.

17. Perez-Padilla JR, Slawinski E, Difrancesco LM, Feige RR, Remmers JE, Whitelaw WA. Characteristics of the snoring noise in patients with and without occlusive apnea. Am Rev Respir Dis 1993; 147: 635-644. 